\title{
ON THE POSSIBILITY OF NONLINEAR QUANTUM EVOLUTION AND SUPERLUMINAL COMMUNICATION
}

\author{
Shan Gao \\ Institute for the History of Natural Sciences, Chinese Academy of Sciences \\ Beijing 100190, People's Republic of China \\ gaoshan@ihns.ac.cn
}

\begin{abstract}
A possible mechanism of nonlinear quantum evolution is introduced and its implications for quantum communication are investigated. First, it is argued that an appropriate combination of wavefunction collapse and the consciousness of observer may permit the observer to distinguish nonorthogonal quantum states in principle, and thus consciousness will introduce certain nonlinearity into quantum dynamics. Next, it is shown that the distinguishability of nonorthogonal states can be used to achieve quantum superluminal communication, by which information can be transmitted nonlocally and faster than the speed of light. Finally, the issue of apparent incompatibility between superluminal communication and special relativity is briefly addressed.
\end{abstract}

Keywords: nonlinear quantum evolution; consciousness; superluminal communication.

\section{Introduction}

It has been known that small nonlinearity in quantum dynamics may have important implications for quantum computation and quantum communication. For example, if quantum states exhibit small nonlinearities during time evolution (see, e.g. Ref. 1), then quantum computers can be used to solve NP-complete problems in polynomial time ${ }^{2}$, and moreover, even though the unconditional security of quantum communication may be broken, a more secure quantum superluminal communication can be achieved. ${ }^{3-5}$ In this paper, we will introduce a possible mechanism of nonlinear quantum evolution and investigate its implications for quantum communication.

\section{A possible way to distinguish nonorthogonal quantum states}

Although quantum mechanics is one pillar of modern physics, it is plagued by the notorious measurement problem, and does not provide a complete description of the measurement process. The main alternatives to a complete quantum theory include dynamical collapse theories ${ }^{6}$, the many-worlds interpretation, and the de Broglie-Bohm theory. The latter two replace the collapse postulate in quantum mechanics with some new structures, such as branching worlds and Bohmian trajectories, while the former integrate the collapse postulate with the normal Schrödinger evolution into a unified dynamics. It has been recently argued that the dynamical collapse theories are probably in the right direction by admitting wavefunction collapse. ${ }^{7,8}$ Here we will mainly present our 
analysis in the framework of dynamical collapse theories ${ }^{\mathrm{a}}$. The analysis only relies on a common characteristic of the theories, i.e., that the collapse of the wave function is an objective dynamical process, essentially independent of the consciousness of observer.

It is a result of quantum mechanics that nonorthogonal quantum states cannot be distinguished. Moreover, it has been shown that nonorthogonal quantum states cannot be distinguished (by physical measuring devices) in dynamical collapse theories either. ${ }^{9}$ However, it has been argued that a conscious being can distinguish his definite perception states and the quantum superpositions of these states, and thus when the physical measuring device is replaced by a conscious observer, nonorthogonal states can be distinguished in principle in dynamical collapse theories. ${ }^{5}$ In the following, we will introduce this result.

Let $\psi_{1}$ and $\psi_{2}$ be the physical states of two definite perceptions of a conscious being, and $\psi_{1}+\psi_{2}$ is the quantum superposition of these two states. For example, $\psi_{1}$ and $\psi_{2}$ are triggered respectively by a small number of photons with a certain frequency entering into the eyes of the conscious being from two directions, and $\psi_{1}+\psi_{2}$ is triggered by the superposition of these two input states. Suppose the conscious being satisfies the following slow collapse condition, i.e., that the collapse time of the superposition state $\psi_{1}+\psi_{2}$, denoted by $t c$, is longer than the conscious time tp of the conscious being for forming the perceptions $\psi_{1}$ or $\psi_{2}$, and the time difference is large enough for him to identify. This condition ensures that consciousness can take part in the process of wavefunction collapse; otherwise consciousness can only appear after the collapse and will surely have no influence upon the collapse process. Now we will explain why the conscious being can distinguish the definite perception state $\psi_{1}$ or $\psi_{2}$ and the superposition state $\psi_{1}+\psi_{2}$.

It is a natural assumption that a definite conscious perception, which is either $\psi_{1}$ or $\psi_{2}$, appears only after the superposition state $\psi_{1}+\psi_{2}$ collapses into $\psi_{1}$ or $\psi_{2}{ }^{b}$. Then the conscious being can have a definite perception after the conscious time tp for the states $\psi_{1}$ and $\psi_{2}$, but only after the collapse time tc can the conscious being have a definite perception for the superposition state $\psi_{1}+\psi_{2}$. Since the conscious being satisfies the slow collapse condition and can distinguish the times tp and $t c$, he can distinguish the definite perception state $\psi_{1}$ or $\psi_{2}$ and the superposition state $\psi_{1}+\psi_{2}{ }^{\mathrm{c}}$.

In fact, we can also give a compact proof by reduction to absurdity. Assume that a conscious being cannot distinguish the definite perception states $\psi_{1}$ or $\psi_{2}$ and the superposition state $\psi_{1}+\psi_{2}$. This requires that for the superposition state $\psi_{1}+\psi_{2}$ the conscious being must have the perception $\psi_{1}$ or $\psi_{2}$ immediately after the conscious time $t p$, and moreover, the perception must be exactly the same as his perception after the collapse of the superposition state $\psi_{1}+\psi_{2}$. Since the conscious time tp is shorter than the

\footnotetext{
${ }^{a}$ Although our analysis may also apply to the other two alternatives ${ }^{5}$, it does not apply to the many-mind interpretation of quantum mechanics and conscious causes collapse theory.

${ }^{\mathrm{b}}$ When this assumption is not true, i.e., when the conscious being in a superposition state can have a definite perception before the collapse has completed, it can still be argued that the conscious being can distinguish the states $\psi_{1}+\psi_{2}$ and $\psi_{1}$ or $\psi_{2}$ with non-zero probability. ${ }^{5}$

${ }^{\mathrm{c}}$ A similar argument was first given in Ref. 10 .
} 
collapse time tc, the requirement means that the conscious being knows the collapse result beforehand. This is impossible due to the essential randomness of the collapse process.

The above result relies on a very stringent condition, the slow collapse condition, which says that for a conscious being the collapse time of a superposition of his definite conscious perceptions is longer than his normal conscious time. Whether this condition is readily available for human brains depends on concrete models of consciousness and wavefunction collapse. However, it should be pointed out that the collapse time of a single superposition state is an essentially stochastic variable, whose value can range between zero and infinity. As a result, the slow collapse condition can always be satisfied in some collapse events with non-zero probability. For these collapse processes, the collapse time of the single superposition state is much longer than the (average) collapse time and the normal conscious time, and thus the conscious being can distinguish the nonorthogonal states. As we will see below, this ultimate possibility may have important implications for quantum communication.

\section{Consciousness and nonlinear quantum evolution}

We have argued that a conscious being can in principle distinguish the nonorthogonal states $\psi_{1}+\psi_{2}\left(\right.$ or $\left.\psi_{1}-\psi_{2}\right)$ and $\psi_{1}$ or $\psi_{2}$, which is an impossible task for a physical measuring system without consciousness. This also means consciousness will introduce one kind of nonlinear quantum evolution. The nonlinearity is definite, not stochastic (cf. Refs. 6, 11). This immediately raises two questions: (1). How can consciousness solve the difficulties of nonlinear quantum mechanics? and (2). Exactly why does consciousness have the superpower to violate the superposition principle? In the following, we will answer these two questions.

It is well known that nonlinear quantum mechanics has a general characteristic, namely that the description of composite systems depends on a particular basis in the system's Hilbert space. This is a serious difficulty for nonlinear quantum mechanics because it makes such theories inconsistent. However, the consciousness of an observer will naturally select a privileged basis in its state space; for physically definite perception states there is a one-to-one correspondence between the physical state and its conscious content, while for superpositions of these definite perception states there is none. In other words, a conscious observer can be aware of the content of physically definite perceptions, but can be aware of none of the content of these perceptions when being in a superposition of them. Thus the nonlinear quantum evolution introduced by consciousness is logically consistent.

Now we turn to the second deeper question. As noted before, a conscious observer can be aware of the change of its perception state, especially the transition from a superposition of different conscious perceptions to one of the conscious perceptions. But a physical measuring device, which is assumed to have no consciousness, cannot record this transition according to quantum mechanics, though it can indeed record some other changes of its physical state such as temperature change etc. Then why is consciousness 
special? The reason is that for a superposition of physically definite perceptions, the physical state is indefinite, while the conscious awareness is always definite, no matter what it is. Even if the observer is conscious of none of the content corresponding to one of the perceptions in the superposition or even nothing, his conscious perception is still definite, which can be distinguished from other conscious perceptions. It is this particular link between physical state and conscious content that introduces the definite nonlinearity in the quantum evolution of the physical state of a conscious observer.

Let's give a concrete example. Suppose the physical state of a conscious observer is $\psi_{1}+\psi_{2}$, and the observer is conscious of being in a superposition state. Then the total state is not $\psi_{1} x_{1}+\psi_{2} x_{2}$, but $\left(\psi_{1} x_{1}+\psi_{2} x_{2}\right) x_{12}$, where $x_{1}, x_{2}$ are the conscious states corresponding to the physical states $\psi_{1}, \psi_{2}$, and $\mathrm{x}_{12}$ denotes the conscious state of the observer who is conscious of being in a superposition. Moreover, since the conscious perception can be put into memory and further lead to some external outputs by verbal report or physical action, the final state will be $\left(\psi_{1} \mathrm{x}_{1}+\psi_{2} \mathrm{x}_{2}\right) \mathrm{x}_{12} \psi_{12}$, where $\psi_{12}$ is the physical state resulting from the conscious state $\mathrm{x}_{12}$. This indicates that consciousness introduces one kind of (definite) nonlinearity to the quantum evolution of the physical states.

The above argument can be summarized in a clearer way. A conscious mental state, unlike a physical state, cannot be an indefinite superposition state. We either have conscious perception $\mathrm{x}$ or have no conscious perception $\mathrm{x}$. We cannot both have conscious perception $\mathrm{x}$ and have no conscious perception $\mathrm{x}$. We know this by selfawareness. In this way, consciousness rejects quantum superposition and introduces one kind of nonlinear quantum evolution.

\section{Implications for quantum communication}

The existence of nonlinear quantum evolution may have important implications for quantum communication. For example, if quantum states exhibit small nonlinearities during time evolution, then superluminal communication can be readily achieved. ${ }^{3-5}$ Here we will give a simple example to illustrate how to realize quantum superluminal communication (QSC) based on the nonlinear quantum evolution introduced by consciousness.

We use polarization-correlated photon pairs as carriers of information. The experimental setting is shown in Fig. 1. Pairs of photons, whose frequencies are $v_{1}$ and $v_{2}$, are emitted in the $-\mathrm{z}$ direction and $+\mathrm{z}$ direction from a source. Then they are analyzed by a single-channel polarizer $\pi_{1}$ and a two-channel polarizer $\pi_{2}$, respectively. Let the sender operate the optical switch $C_{1}$ and the receiver read the output of NSDD (Nonorthogonal States Distinguishing Device), a device that can distinguish nonorthogonal photon states. The communication protocol is as follows. The encoding rule for the sender is that not measuring the photon represents sending the code ' 0 ', and measuring the photon represents sending the code ' 1 '. The decoding rule for the receiver is: when the photon enters NSDD from one direction, the device outputs ' 0 ', representing having received the code ' 0 ', and when the photon enters NSDD from two directions at the same time, the device output ' 1 ', representing having received the code ' 1 '. 


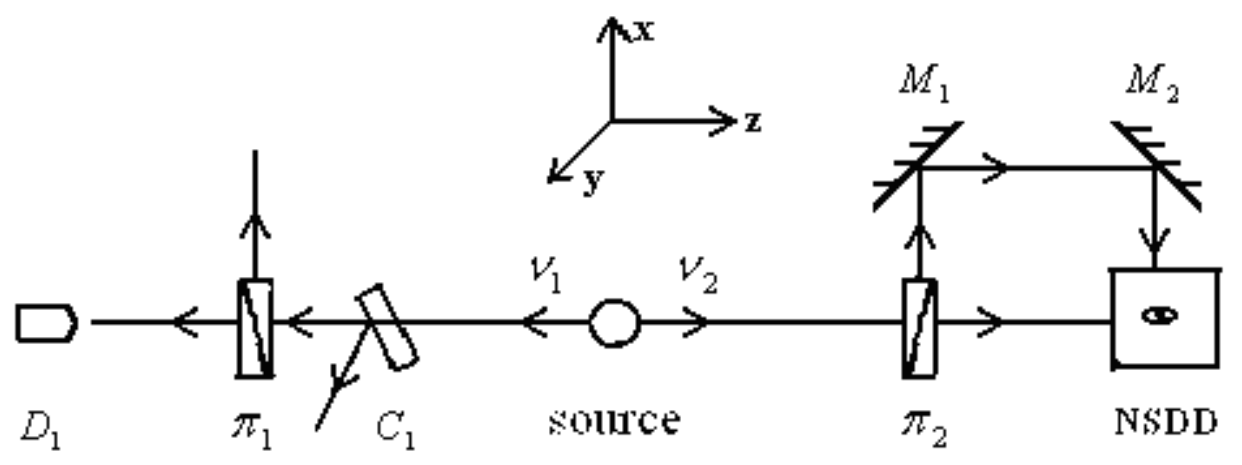

Fig. 1. The setting of a QSC scheme

The communication process can be described as follows. When the sender wants to send a code ' 0 ', he controls the optical switch $C_{1}$ to let the photon $v_{1}$ move freely and not be analyzed by the polarizer $\pi_{1}$. Then the photon $v_{2}$ is in a superposition of two directions after it passes the polarizer $\pi_{2}$, and it enters NSDD from two directions at the same time (in one direction it is reflected by two mirrors $M_{1}$ and $M_{2}$ ). Thus the output of NSDD is ' 0 ', and the receiver can decode the sent code as ' 0 '. When the sender wants to send a code ' 1 ', he controls the optical switch $C_{1}$ to allow the photon $v_{1}$ to be analyzed by the polarizer $\pi_{1}$ and detected by $D_{1}$ before the photon $v_{2}$ arrives at NSDD. Then the state of the photon $v_{2}$ collapses to a state with definite direction, and it enters NSDD from one direction. Thus the output of NSDD is ' 1 ', and the receiver can decode the sent code as ' 1 '. In this way, the sender and the receiver can achieve superluminal communication.

The most important component of the above QSC setting is NSDD. The device can be implemented through directly using a conscious being (as argued before) or an advanced self-awareness simulator that may be available in the future. Since it is difficult for a conscious being like us to perceive a single photon, the superposition states of a small number of photons may be needed to achieve QSC in practical situations.

It can be seen that the communication rate of QSC is limited by the perception time of the conscious being ${ }^{\mathrm{d}}$. An optimizing method is to combine QSC and quantum teleportation. For quantum teleportation, the information transferred via the classical channel is very little, and the majority of information is transferred through the quantum channel. Thus, by replacing the classical communication with QSC, quantum teleportation will become superluminal communication and the communication rate may be largely increased.

QSC will have several advantages over conventional communications. First, its transfer delay is independent of the communication distance, and can be zero in principle. Thus QSC is truly real time communication, no matter how far the communicating parties are separated. Secondly, when the carriers of information are stored in the

\footnotetext{
${ }^{d}$ The perception time of our human beings is of the order of $0.1 \mathrm{~s}$, thus the corresponding communication rate of superluminal communication will be only of the order of $10 \mathrm{bps}$.
} 
communicating parties, QSC is not influenced by the environment between them. Thus it is absolutely anti-jamming. Thirdly, due to the same reason, a third party cannot eavesdrop. Thus QSC is also the most secure way to communicate.

Lastly, we give a brief comment on the apparent incompatibility between QSC and special relativity. It has been widely argued that special relativity and quantum mechanics are incompatible when considering the existence of quantum nonlocality, and a preferred Lorentz frame may be needed for describing quantum nonlocal processes such as wavefunction collapse. Once there is a preferred Lorentz frame, QSC will not lead to causal loops and may be consistent. Moreover, if the above scheme of QSC is indeed valid, then it can also be used to determine the preferred Lorentz frame.

\section{Conclusions}

In this paper, we have argued that an appropriate combination of wavefunction collapse and the consciousness of observer may permit the observer to distinguish nonorthogonal quantum states in principle. This means that the consciousness of observer, which has not been seriously taken into account in physics (see, however, Ref. 11), will introduce certain nonlinearity into quantum dynamics. Moreover, we have shown that the distinguishability of nonorthogonal states can be used to achieve quantum superluminal communication, by which information can be transmitted nonlocally and faster than the speed of light. The issue of apparent incompatibility of superluminal communication with special relativity has also been briefly addressed.

\section{References}

1. S. Weinberg, Phys. Rev. Lett. 62, 485 (1989).

2. D. S. Abrams and S. Lloyd, Phys. Rev. Lett. 81, 3992 (1998).

3. N. Gisin, Phys. Lett. A 143, 1 (1990).

4. J. Polchinksi, Phys. Rev. Lett. 66, 397 (1991).

5. S. Gao, Found. Phys. Lett. 17, 167 (2004).

6. G. C. Ghirardi, Collapse Theories, The Stanford Encyclopedia of Philosophy (Winter 2011 Edition), Edward N. Zalta (ed.). (2011).

7. S. Gao, Int. J. Theo. Phys. 45, 1943 (2006).

8. S. Gao, Interpreting Quantum Mechanics in Terms of Random Discontinuous Motion of Particles. http://philsci-archive.pitt.edu/9589/. (2013). Chap.4.

9. G. C. Ghirardi, R. Grassi, J. Butterfield, and G. N. Fleming, Found. Phys. 23, 341 (1993).

10. E. Squires, Phys. Lett. A 163, 356 (1992).

11. E. P. Wigner, Symmetries and Reflections (Indiana University Press, Bloomington, 1967). pp.171-184. 